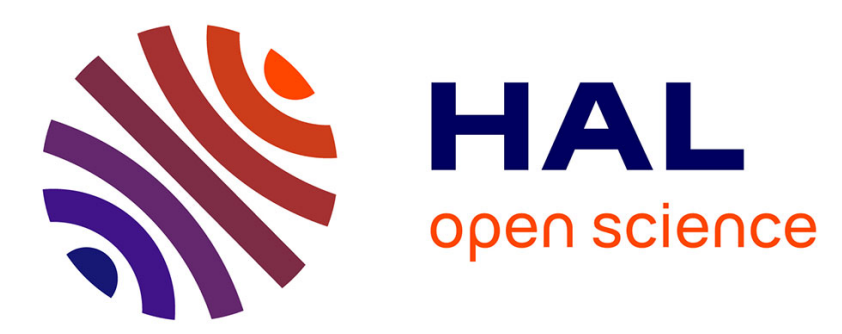

\title{
Cognitive impact of Social Robots: How anthropomorphism boosts performance
}

Nicolas Spatola, Sophie Monceau, Ludovic Ferrand

\section{To cite this version:}

Nicolas Spatola, Sophie Monceau, Ludovic Ferrand. Cognitive impact of Social Robots: How anthropomorphism boosts performance. IEEE Robotics and Automation Magazine, 2020, 27 (3), pp.73-83. 10.1109/MRA.2019.2928823 . hal-02347083v3

\section{HAL Id: hal-02347083 \\ https://hal.science/hal-02347083v3}

Submitted on 22 Sep 2020

HAL is a multi-disciplinary open access archive for the deposit and dissemination of scientific research documents, whether they are published or not. The documents may come from teaching and research institutions in France or abroad, or from public or private research centers.
L'archive ouverte pluridisciplinaire HAL, est destinée au dépôt et à la diffusion de documents scientifiques de niveau recherche, publiés ou non, émanant des établissements d'enseignement et de recherche français ou étrangers, des laboratoires publics ou privés. 


\section{Cognitive impact of Social Robots: How anthropomorphism boosts performance}

\author{
Spatola Nicolas \\ Laboratoire de Psychologie Sociale et \\ Cognitive, CNRS, UMR 6024 \\ Université Clermont Auvergne \\ Clermont-Ferrand, France \\ nicolas.spatola@uca.fr
}

\author{
Monceau Sophie \\ Laboratoire de Psychologie Sociale et \\ Cognitive, CNRS, UMR 6024 \\ Université Clermont Auvergne \\ Clermont-Ferrand, France \\ sophie.monceau@uca.fr
}

\author{
Ferrand Ludovic \\ Laboratoire de Psychologie Sociale et \\ Cognitive, CNRS, UMR 6024 \\ Université Clermont Auvergne \\ Clermont-Ferrand, France \\ ludovic.ferrand@uca.fr
}

\begin{abstract}
There is evidence that selective attention mechanisms in humans can be impacted in performance contexts involving the presence of robotic agents compared to contexts in which they are alone. However, the question of whether this process is due to anthropomorphism attribution, potentially explaining why robots trigger the same effect as humans, remains unclear. We investigated this issue using a selective attention task in a social presence paradigm. One group of participants performed the so-called Eriksen Flanker task in the presence of a robot after a verbal social interaction (i.e., social robot condition), while the other group did the same with a robot that they only described (i.e., non-social robot condition). Results showed that after social interaction, the robot was perceived as having human traits (according to the humanization and anthropomorphism scale). Furthermore, we found a social presence effect (i.e., an improvement in selective attention performance) only in the presence of the social robot but not in that of the non-social one. Finally, this latter effect was mediated by anthropomorphism attributions. Our results suggest that the influence of robot presence is socio-cognitive in nature and that anthropomorphism has a role in the robot presence effect. Theoretical implications are further discussed.
\end{abstract}

Keywords-Social Robotics, Human-Robot Interaction, Selective attention, Anthropomorphism, Eriksen Flanker task, Social presence

\section{INTRODUCTION}

Humanoid robots might become increasingly present in the lives of millions of people worldwide [1], [2]. While tremendous progress has been made in robotics science, the influence that these new humanoid robots may have on human cognition itself remains poorly understood. There is evidence [3]-[6] that the presence of a humanoid robot can lead to effects similar to those caused by human presence in terms of feelings [7] and task performance [3], [4]. However, these pioneering efforts have overlooked both the interaction between attentional processes and the anthropomorphic inferences that may be involved in the influence of robotic presence. Here, we take advantage of research on human presence and attention and argue that the presence of humanoid robots - even passive-may affect attentional processes, at least when the robot present is anthropomorphized to some extent.

\section{A. Brief review of earlier research on social presence and social robot presence effects}

A century of research in experimental social psychology has shown a tendency for humans to perform differently on a myriad of motor and cognitive tasks when in the presence of conspecifics - other members of the same species - than they do when they are alone. Zajonc [8] was the first to notice that the presence of observers or coactors typically facilitates performance on easy or well-learned tasks, and impairs performance on difficult or poorly-learned tasks. Taking this idea further, Baron's distraction/conflict theory suggested an integrative attentional view of this so-called Social Facilitation/Inhibition Effect (SFI) [9]. The key idea was that social presence, when it is distracting or diverts attention away from the focal task, can create attentional conflict, namely a form of response conflict concerning the appropriate attentional response (pay attention to the focal task or to the person present). This conflict, in turn, may result in cognitive overload and, ultimately, restrict the attentional focus.

Attentional focusing may produce different effects. Firstly, through the activation of dominant responses. In this case, the process facilitates performance. Because of the smaller amount of cognitive resources available, peripheral stimuli are screened out when the task is simple or requires attention to be paid to a small number of central cues. Secondly, through the impairment of performance (by neglecting certain crucial stimuli) when the task is more complex or demands attention to a wide range of cues.

According to the Ethopoeia concept [10], automatic social reactions are triggered by situations that include social cues, which need not consist solely of other humans [11], [12]. This concept also seems to apply to HumanRobot Interactions (HRI) [3], [5], [7], [10], [13]. For example, it has been shown that the modalities of interaction might directly impact how humans consider artificial social agents in response to one and the same robot [3], [5], [6].

For instance, a recent study [5] showed that a robot acting positively with participants (e.g., with empathy and consideration) during a question/answer paradigm produced less SFI than a robot acting negatively (e.g., with contempt and a lack of empathy). In the presence of a "bad" robot, participants tended to improve their selective attention performances (i.e., the ability to select the pertinent information in a task or a context and to inhibit the nonrelevant information) on the Stroop task (in which individuals have to identify the color in which a word is printed, while ignoring the word itself). No such improvement was observed in the presence of the "good" robot. As has also been found in human-human studies [14], [15], the presence of a robot seems, under certain circumstances, to improve the selectivity of attention to relevant information at the expense of competing cues in easy tasks [14]. Indeed, in Spatola et al.'s experiment, the bad social robot had the same impact on selective attention performance as human presence [14]. The authors suggested that this could have been due either to the unexpected activation of behavior due to the attribution of anthropomorphic traits (i.e., the attribution of human 
characteristics to a non-human) [16] or to the perceived evaluative threat associated with the presence of an unpleasant robot [17]. However, while Spatola et al.'s study was informative, robots designed to live with us are not intended to appear threatening. Nevertheless, their impact on attentional mechanisms and behavior in general may actually depend to a great extent on what people come to believe (anthropomorphic inferences) about them. Subsequent research has shown that SFI effects may occur following a previous social interaction with a robot. This is due to the emergence of anthropomorphization processes triggered by the situation [6]. Taken together, these findings run counter to a purely mechanistic approach that reduces the effects of a robot's presence to the action of physical visual or acoustic distraction by an object, which may sometimes impact human performances (see also [9]), and to the role of anthropomorphism.

However, because of the design used, Spatola et al.'s [5], [6] research could not specify the exact role of anthropomorphic inferences in the Robot SFI on cognitive performance and the nature of the anthropomorphic mediator of SFI effects remains unclear. Indeed, anthropomorphism is a complex concept that brings together various psychological processes such as social categorization or the modulation of the conceptual distance between humans and robots. Finally, this research indicates that the presence of a social robot can boost attentional focusing even when this process requires the deployment of inhibitory control. However, this conclusion is limited solely to the task used in the study in question, namely the Stroop task.

Regarding the incentive to introduce robots in various environments such as school [18], it seems of prime importance to gain a thorough understanding of the impact of robot presence on human cognitive processes. One of the basic processes for learning is the selective attention process that helps to filter out incidental irrelevant information and focus on the information/stimuli that demand attention. Here, we intend to compare the SFI-mediating role of two major psychosocial mechanisms involved in interpersonal perception and behavior in a new attentional control task, the Flanker task, which measures selective attention performances. We will therefore focus our research on the following questions:

First, will Robot SFI be found in another gold-standard task of response inhibition measuring selective attention? Second, are SFI effects involving robots due to the use of universal social perceptual dimensions that drive social categorization (i.e., warmth and competence) by the observer [19]. Third, are SFI effects involving robots also based on the attribution of concepts that are uniquely inherent to humans [20] and thus to a modulation of the conceptual distance between the robot and the representation of what humans are? Finally, are these processes mutually exclusive?

\section{B. The Flanker task}

To generalize the Robot SFI, we used the Eriksen Flanker task (EFT) [21], [22]. The EFT requires participants to judge the direction of the central cue in multi-component stimuli. The task comprised congruent (e.g. $\gg>>>)$ and incongruent stimuli (e.g. $\langle<><<$ ) that were used to assess the ability to suppress information that is not relevant for the task that is to be performed. Response times are usually longer on incongruent than on congruent trials because of the incompatibility between the peripheral items and the central cue (i.e., the target). On incongruent trials, the interference caused by contradictory irrelevant information requires a supplementary inhibition process, which is not present on congruent trials. Thus, the EFT specifically makes it possible to measure selective attention performance. As in the Stroop task, the response inhibition processes involved in the EFT are sensitive to the social nature of the context and the presence of other fellow creatures [22], [23].

\section{The perception of social robots}

The "Computers Are Social Actors" theory [24] posits that people may understand and relate to machines in a similar way that they do to their fellow creatures. Humans do indeed tend to apply the same social scripts (specifying actions to be produced in various social situations [25] such as human-robot or human-human interactions [10]). This tendency may be strengthened by the physical presence of the artificial agent and its humanoid shape, as this shape provides more social cues to the observer [26]-[29].

Interestingly, this process may result in two, nonexclusive, socio-cognitive phenomena, 1) the use of universal dimensions of social perception to apply social categorizations to artificial agents [30], [31] and 2) the change in the intrinsic representation of robots as a modulation of the conceptual human-robot distance [6], [20]. Both processes are related to how people perceive and consider others but also to how they behave in their presence [32]-[34].

1) The use of universal dimensions of social perception to characterize artificial agents

According to Fiske, Cuddy and Glick [19], [35], most interpersonal impressions are distributed along two main dimensions: warmth (e.g., sincerity, trustworthiness, morality) and competence (e.g., ambition, confidence). The warmth dimension predicts active behaviors such as helping (high warmth) or attacking (low warmth). The competence dimension predicts passive behaviors such as association (high competence) or neglect (low competence). The model is based on the notion that, when interacting with an individual, people will assess their intent to either help or harm (i.e., warmth dimension) and their competence/capacity to act accordingly (i.e., competence dimension). The results of this evaluation will trigger a social categorization process, which is the process of classifying people into groups based on similar characteristics. For example, members of social groups stereotyped as warm and competent are perceived much more positively than members of social groups stereotyped as cold and incompetent. Previous research indicates that social categorization processes underlying the perception of persons can be generalized to robots [31], [36]. Inspired by Fiske et al.'s model, Carpinella et al. have proposed a questionnaire to assess these dimensions of social evaluation with regard to robots [30]. We will therefore use this tool to evaluate the scope of the mediating role of the warmth and competence dimensions in Robot SFI.

\section{2) The change in the intrinsic representation of robots}

The theory of dehumanization describes a disposition towards others in which the observer deprives the other of 
social or fundamental human characteristics. The dehumanization process, theorized by Haslam [20], refers to perceiving or treating people as more or less human. This process consists of two bi-dimensional negative/positive constructs illustrating the human essence: 1) animalistic dehumanization opposed to human uniqueness (e.g., Amorality / Moral sensibility), which distinguishes humans from other animals on typical socially learned characteristics; 2) mechanistic dehumanization opposed to human nature (e.g., Rigidity / Cognitive openness), which represents fundamental characteristics held inherently since birth central to human beings and the distance between machines and humans. Interestingly, these dimensions echo the warmth/competence concepts. However, the dehumanization process is based on a modulation of the distance between the representation of what defines the concept of Human and the representation of the other [34], [37], [38], rather than on a stereotyping process [39]. Harris and colleagues suggested that dehumanization occurs due to the decrease of neural activity towards the target [40]. Interestingly, this difference in neural activity is also observed in the comparison between Human-Human and Human-Robot interactions [41], [42]. This idea is supported by fMRI results showing that HRI is underpinned by human-human neuro-social mechanisms [42]-[44].

Regarding HRI, this process seems to be both bottom-up and top-down. First, perception (i.e., a bottom-up process) activates a motor resonance process. Motor resonance makes it possible to directly and immediately understand an action performed by others by providing a motor representation of the observed action through which the observer's motor system enters a state of direct resonance with that of the agent [45]-[47]. In the same way that we cannot refrain from recognizing a face or a word when we perceive it, we cannot help but represent in motor terms the actions we perceive. During interactions with humans, the information that leads to this activity is integrated completely automatically [48], [49] and is resistant to modulation by context [50], [51]. This system could be used to anticipate other people's actions [52]. In the case of robots, this automatic need to anticipate is still present [53]. However, the resonance would naturally be weaker and more context-dependent [54]. The reason could lie in the fact that it is more difficult to match the representation of a specific human action with the actual action of the robot than it is to match it with the actual action of another human [55]. If the context requires the system to interpret the movements of the robot in terms of human characteristics then the system could act more forcefully to transform a weak bottom-up signal into a valid representation at the level of motor resonance by using top-down inferences concerning robots (i.e., social scripts, anthropomorphic inferences) [53], [56]. Due to these bottom-up/top-down processes, robots could trigger the same effect as humans do [3], [5] simply by being present and could, for example, activate "humanization" processes (i.e., attributing to them intentions, emotions or mental states comparable to those of humans) [44], [57]-[59]. In other words, the process might represent the opposite of dehumanization. Furthermore, because in HRI this process is sensitive to contextual pressures, one and the same robot will not be considered to be the same in different contexts (e.g., social vs. non-social context) and will produce different socio-cognitive effects [6].

\section{THE PRESENT STUDY}

\section{A. Objective}

In this study, our aim is to evaluate the mediating role on SFI of the universal social perceptual dimensions of warmth and competence, as well as of the intrinsic representation of robots activated, within a social HRI compared to a nonsocial HRI. To do so, we used a verbal social interaction paradigm with a simple humanoid robot without any facial expression in order to control for emotional priming effects. Verbal social HRI is thought to increase the likelihood of human characteristics being attributed to the robot because of the activation of automatic social scripts that we use in human-human interactions [10]. This design should therefore maximize anthropomorphic inferences in only one of two robotic conditions [6]. After the HRI induction, the participants performed a selective attention task in the passive presence of the robot.

\section{B. Hypotheses}

First, we hypothesized that after a social HRI, robots should trigger the same SFI on selective attention as humans do, that is to say an improvement in response inhibition performances [3], [5], [14], [15]. Because the inhibition process is similar in both the Stroop task and the EFT, we hypothesized that in the presence of the social robot, participants should produce better performances than in the presence of the non-social robot. The presence of the social robot should result in the attentional focus being narrowed down to the central cue at the expense of the peripheral cues, thereby reducing the response conflict [6], [9].

Second, we expected to observe an increased level of attribution on both the universal dimensions of warmth and competence, as well as an increase in the intrinsic representation of robots on essential human dimensions. More specifically, we expected the robot in the social robot condition to be attributed a higher level of warmth due to the social nature of the experience, and to be attributed more competence due to the more complex HRI in the social condition compared to the simple description required in the non-social condition. Regarding the intrinsic representation of robots, we expected the participants to perceive the robots as sharing more human nature traits in the social than in the non-social condition.

Third, the SFI effect in the social condition should be mediated by the inferences made about the robot. Indeed, according to previous research showing that social interaction promotes the attribution of human traits [6], [60], we hypothesized that the more highly the participants perceived the robot on the universal dimensions of warmth and competence used in social categorization, the more sensitive they should be to its passive presence, thus resulting in a higher SFI. The same phenomenon should occur as a function of the level of perceived shared human traits.

Finally, we will conduct an exploratory analysis to compare the hypothetical mediation factor of SFI. The purpose is to identify the main determinant of this sociocognitive phenomenon. 


\section{METHOD}

\section{A. Participants}

The participants were 80 French students at Université Clermont Auvergne (Mage $=19.32$ years, $\mathrm{SD}=2.05,68$ females and 12 males) with normal (or corrected-to-normal) vision (27 in the Alone condition, 27 in the Non-Social Robot condition, and 26 in the Social Robot condition). Sample size was determined-as recommended by Tabachnick and Fidell [61] — on the basis of the desired power (.80), alpha level (.05), number of groups (three in the main analysis), and anticipated effect size based on human presence effects (using between-subjects design) in a Stroop paradigm $\left(\eta^{2}{ }_{p}=.40\right.$; [23]). Using $G^{*}$ Power 3.1 [62], the minimum required sample size was calculated as 66 .

\section{B. Procedure}

The participants performed the Eriksen Flanker task twice (Session 1, Session 2). First (Session 1), all the participants performed the task alone (the experimenter left the room). The first session was used as a control to ensure that the different groups performed equally on the Flanker task without any experimental induction. It also made it possible to ensure that all the participants had comparable knowledge and skill with regard to the task. At the end of the first session, the participants were randomly assigned to one of the three conditions:

- In the alone condition, they had to describe a picture of Central Park. This task was used as a distractor task in order to maintain the reliability of group comparisons at the level of intersession activities.

- In the non-social robot condition, they had to describe the design of the robot that the experimenter brought into the room.

- Finally, in the social robot condition, they were asked to interact verbally with the same robot in (unknown to them) a "Wizard of Oz paradigm" [63]. The robot was presented as a work in progress. The robot was the same in both the social and non-social conditions.

These two conditions have been previously pretested (see [6]).

The participants were asked whether they agreed to describe or to interact with the robot, according to the experimental condition. They were told that the purpose of the study was to collect their impressions on the robot in order to improve it. The tasks took the same amount of time.

After a break, all the participants performed the Flanker task again, either alone (as before) or in the presence of the robot. In the two robot presence conditions, the robot was positioned in front of the participants and watched them for $60 \%$ of the time (for a similar procedure with human presence, see [14], [15]). The interaction always followed the same pre-established script (See Supplementary materials on OSF) (Figure 1).

\section{Wizard of $\mathrm{Oz}$}

A smartphone with a Bluetooth connection controlled the robot, a Meccanoid G15 KS. A Motorola Moto G 4G controlled the movements. The operator produced the robot's speech using a modified voice. The voice was designed using the Pixie voice module on Voxal from $\mathrm{NCH}$ Software. A hidden control camera was used to ensure correct control of movements and responses in the Wizard of Oz paradigm [5], [63].

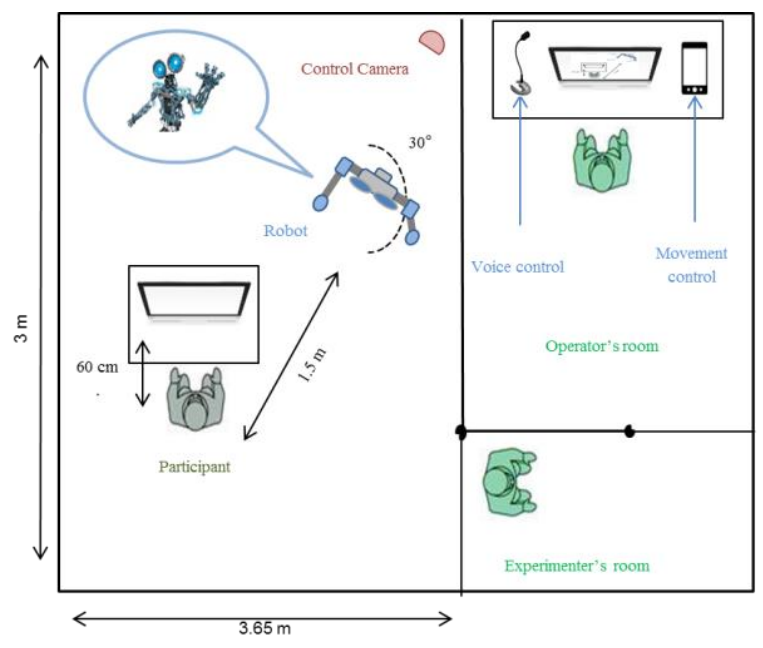

Fig. 1. Experimental setting.

\section{Eriksen Flanker Task}

Each session (session 1 and 2) consisted of 220 trials, i.e. 110 congruent $(55$ “<<<<", and 55" $>>>>>)$ and 110 incongruent (55 " $<<><<"$, and 55 " $>><>>$ ") trials. The Flanker interference was computed by subtracting the response times (RT) of congruent trials from the RTs of incongruent trials (in $\mathrm{ms}$ ). The higher the score, the less efficient selective attention performance was.

Before the experimental trials in the first session, the participants responded to 20 samples to enable them to learn the response keys (“\#\#<\#\#”, “\#\#>\#\#”).

\section{E. Anthropomorphic inferences.}

At the end of the experiment, the participants completed the Robotic Social Attribute Scale [30]. This scale makes it possible to evaluate robots on the dimensions of warmth (e.g. "emotional"), competence (e.g. "interactive") and discomfort (i.e. "I find this robot scary"). This scale has been standardized to measure the social perception of robots based on their appearance. For each dimension, the participants had to indicate whether they thought the different characteristics fitted the presented robot (from 1 "does not fit at all" to 5 "totally fits").

The participants also completed the humanness scale based on Haslam's dehumanization taxonomy [20], which consists of four dimensions: human uniqueness (e.g., moral sensibility), animalistic dehumanization (e.g., irrationality), human nature (e.g., interpersonal warmth), and mechanistic dehumanization (e.g., inertness). Once again, for each dimension, the participants rated (on a scale of 1 to 5) whether or not they would attribute the related characteristics to the robot that was present with them. For a similar use of these questionnaires, see [5].

We presented the scale at the end of the study to avoid any priming effect due to questionnaires. However, the anthropomorphic inferences in conditions with and without robot interaction had been evaluated in a pre-test, see [6]. 
TABLE I. MEAN CORRECT RESPONSE TIMES (IN MILLISECONDS), STANDARD ERRORS (IN PARENTHESES) AND ERROR RATES AS A FUNCTION OF THE TYPE OF STIMULUS, FLANKER SESSION, AND EXPERIMENTAL CONDITION

\begin{tabular}{|c|c|c|c|c|c|c|c|}
\hline & & \multicolumn{2}{|c|}{ Alone } & \multicolumn{2}{|c|}{ Non-social robot } & \multicolumn{2}{|c|}{ Social robot } \\
\hline & & Session 1 & Session 2 & Session 1 & Session 2 & Session 1 & Session 2 \\
\hline \multirow{2}{*}{ Congruent } & $\mathrm{RT}$ & $246.61(13.21)$ & $220.36(12.14)$ & $275.72(13.21)$ & $229.22(14.96)$ & $247.72(13.80)$ & $231.72(12.69)$ \\
\hline & ER & $.95(.01)$ & $.97(.01)$ & $.96(.01)$ & $.98(.01)$ & $.95(.01)$ & $.95(.01)$ \\
\hline \multirow{2}{*}{ Incongruent } & RT & $351.86(22.57)$ & $296.83(14.96)$ & $368.05(22.57)$ & $310.11(14.96)$ & $338.85(23.57)$ & $287.76(15.62)$ \\
\hline & ER & $.87(.03)$ & $.91(.02)$ & $.87(.01)$ & $.88(.02)$ & $.81(.03)$ & $.83(.02)$ \\
\hline \multirow{4}{*}{$\begin{array}{c}\text { Flanker } \\
\text { Interference }\end{array}$} & RT & $105.25(18.17)$ & $76.47(8.01)$ & $92.34(18.17)$ & $80.98(8.01)$ & $91.13(18.98)$ & $51.04(8.37)$ \\
\hline & Results & $\begin{array}{l}\mathrm{p}<.001 \\
\eta_{p}^{2}=.26\end{array}$ & $\begin{array}{l}\mathrm{p}<.001 \\
\eta_{p}^{2}=.58\end{array}$ & $\begin{array}{l}\mathrm{p}<.001 \\
\eta_{p}^{2}=.28\end{array}$ & $\begin{array}{l}\mathrm{p}<.001 \\
\eta_{p}^{2}=.60\end{array}$ & $\begin{array}{l}\mathrm{p}<.001 \\
\eta_{p}^{2}=.26\end{array}$ & $\begin{array}{l}\mathrm{p}<.001 \\
\eta_{p}^{2}=.36\end{array}$ \\
\hline & ER & $.08(.03)$ & $.06(.02)$ & $.10(.03)$ & $.10(.02)$ & $.14(.03)$ & $.12(.02)$ \\
\hline & Results & $\begin{array}{l}\mathrm{p}=.007 \\
\eta_{p}^{2}=.11\end{array}$ & $\begin{array}{l}\mathrm{p}=.004 \\
\eta_{p}^{2}=.12\end{array}$ & $\begin{array}{l}\mathrm{p}=.001 \\
\eta_{p}^{2}=.16\end{array}$ & $\begin{array}{l}\mathrm{p}<.001 \\
\eta_{p}^{2}=.23\end{array}$ & $\begin{array}{l}\mathrm{p}<.001 \\
\eta_{p}^{2}=.26\end{array}$ & $\begin{array}{l}\mathrm{p}<.001 \\
\eta_{p}^{2}=.30\end{array}$ \\
\hline
\end{tabular}

\section{RESULTS}

\section{A. Preliminary analyses.}

The data from three participants were excluded from the analysis because of technical problems (i.e., robot disconnection, program crash). In addition, the data from participants with an accuracy rate less than $70 \%$ were discarded because we could not be sure that they performed the task conscientiously ( 7 participants). The results obtained from the remaining participants are summarized in Table 1. Errors occurred on $8.83 \%$ of the trials and were analyzed independently. Correct trials with reaction times (RT) more than 2.5 standard deviations above or below the mean in each condition and for each participant were considered outliers and removed from the RT analyses. These amounted to 771 out of 26,805 trials $(2.87 \%$ of the trials). This filtering procedure has the advantage of excluding extreme values without specifically affecting the data of any one condition or any one participant (raw data are available on OSF). The analyses were conducted using SPSS 24 and the mediation analyses using the PROCESS plug-in.

\section{B. Control session.}

To ensure that all the groups were comparable, we conducted two repeated-measures ANOVAs, on both errors and RTs in Session 1, including the type of stimulus (congruent vs. incongruent) as within factor and the experimental condition as between factor. Results showed no significant interaction between experimental condition and type of stimulus on either errors, $F(2,67)=1.25, p=$ $.293, \eta^{2} p=.04$ or RTs, $F(2,67)=.021, p=.979, \eta^{2} p<.01$. However, the participants were more accurate on congruent trials than incongruent trails, $F(1,67)=41.84, p<.001, \eta^{2} p$ $=.38,95 \% C I[.07 ; .14]$; and responses on congruent trials were faster than on incongruent trials, $F(1,67)=71.82, p<$ $.001, \eta^{2} p=.52,95 \%$ CI $[68.99 ; 111.49]$.

\section{Experimental session.}

We again conducted a repeated-measures ANOVA on Session 2 errors and RTs, including the type of stimulus (congruent vs. incongruent) as within factor, and experimental conditions as between factor. Results showed no interaction effect on errors between the type of stimulus and the experimental condition, $F(2,67)=1.65, p=.201$, $\eta^{2} p=.05$. However, the main effect of the type of stimulus was significant, $F(1,67)=54.70, p<.001, \eta^{2} p=.45$, $95 \% C I[.07 ; .12]$. The same effect was present on RTs, $F(1$, $67)=219.05, p<.001, \eta^{2} p=.77,95 \% C I$ [60.10; 78.84]. Interestingly, we found a type of stimulus by experimental condition interaction on RTs, $F(2,67)=3.82, p=.027, \eta^{2} p$ $=.10,95 \% C I[.07 ; .12]$ (Figure 2). To test our hypothesis, we conducted a planned comparison analysis. This type of analysis reduces the risk of making a Type II error. Results showed that there was no significant difference between the alone and the non-social robot experimental conditions, $t(67)=.39, p=.697, \eta^{2} p<.01$, while the participants in the social robot condition achieved higher selective attention performances than the average of those in the other two conditions, $t(67)=2.74, p=.008, \eta^{2} p=.14$. This confirms our Robot SFI effect hypothesis. The main effect of group on RTs (without taking account of the type of stimulus) was not significant, $F(2,67)=.24, p=.789, \eta^{2} p=.01$.

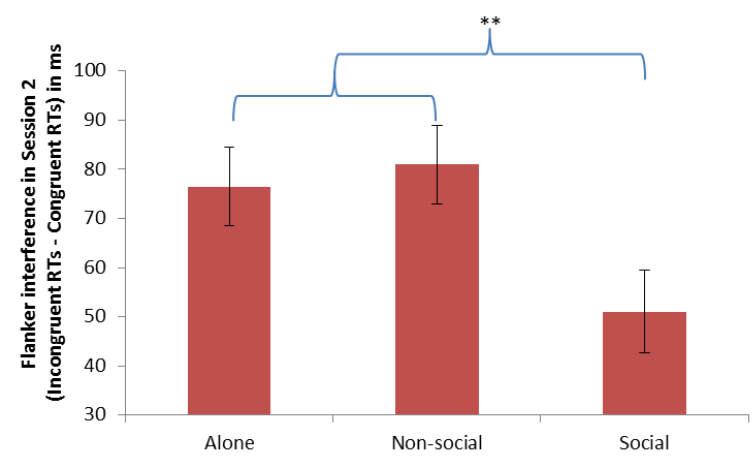

Fig. 1. Eriksen Flanker interference (RTs on congruent trials minus RTs on incongruent trials) as a function of the experimental session (Alone, Non-Social Robot, Social Robot): the less positive the value, the better the selective attention performance. Error bars represent 1 standard error.

\section{Anthropomorphic and humanization attributions.}

If we consider the experimental conditions (social vs. non-social), a MANOVA including the anthropomorphism (i.e., warmth, $\alpha=.82$; competence, $\alpha=.88$; discomfort, $\alpha=$ .77) and humanization (i.e., human uniqueness, $\alpha=.88$; animal dehumanization, $\alpha=.60$; human nature, $\alpha=.77$; mechanistic dehumanization, $\alpha=.67$ ) attribution scores showed that the participants in the social robot condition perceived the robot as having more uniquely human traits (e.g. maturity, logic), $F(1,44)=4.33, p=.043, \eta^{2} p=.09$, $95 \%$ CI $[.04 ; 2.29]$, traits relating to human nature (e.g. cognitive openness), $F(1,44)=6.70, p=.013, \eta^{2} p=.13$, 
95\%CI [.28; 2.24], and warmth traits (e.g. happiness), $F(1,44)=4.33, p=.012, \eta^{2} p=.13,95 \% C I[.27 ; 2.12]$ than those in the non-social robot condition. This finding validates our second hypothesis about the impact of social interaction on robot perception.

\section{1) Human uniqueness}

We conducted a first mediation analysis including experimental conditions (IV), human uniqueness attribution (mediator) and selective attention performance in the second session (DV) in a model, $R^{2}=.39, F(2,43)=13.46, p$ $<.001$. Results showed that the participants attributed more human uniqueness traits to the robots in the social than in the non-social condition, (a1) $t(44)=2.08, p=.043,95 \% C I$ $[.036 ; 2.29]$. When the effect of human uniqueness attribution on selective attention performance was controlled for, (b1) $t(44)=-4.39, p<.001,95 \% C I[-18.64 ;-6.91]$, the direct effect of experimental conditions on selective attention performance, (c) $t(44)=-2.33, p=.025,95 \% C I[-$ $55.72 ;-3.98]$, became non-significant, (c') $t(44)=-1.33, p$ $=.192,95 \% C I[-37.80 ; 7.80]$. This suggests that mediation took place. In sum, in the social robot condition, participants attributed more human uniqueness traits to the robot than they did in the non-social robot condition. After the social interaction, the more uniquely human characteristics they perceived the robot as possessing, the greater the performance improvement generated by the presence of the robot was, $b=-.33,95 \% C I[-.64 ;-.01]$ (Figure 3).

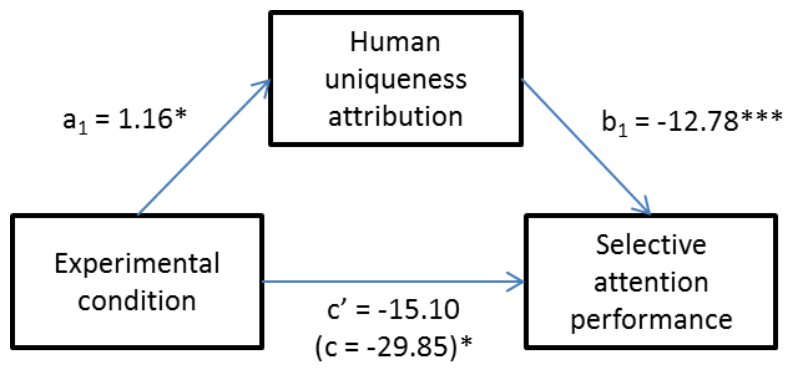

Fig. 3. Mediation of the robot presence effect on standard selective attention performance by human uniqueness inferences.

\section{2) Human nature}

The second mediation analysis differs only in that human nature attribution replaces human uniqueness attribution as a mediator in the model, $R^{2}=.30, F(2,43)=$ $9.33, p<.001$. In confirmation of our hypothesis, the analysis showed that the participants attributed more human nature traits to the robot in the social HRI condition, (a1) $t(44)=2.59, p=.013,95 \% C I[.28 ; 2.24]$. When the effect of human nature attribution on selective attention performance, (b1) $t(44)=-12.29, p=.001,95 \% C I[-19.47 ;-5.11]$, was controlled for, the direct effect of experimental condition on selective attention performance, (c) $t(44)=-2.33, p=.025$, $95 \%$ CI [-55.72; -3.98], became non-significant, (c') $t(44)=$ $-1.17, p=.250,95 \% C I[-39.24 ; 10.50]$, thus suggesting that mediation occurred. Once again, the participants in the social robot condition attributed more human uniqueness traits to the robot than those in the non-social robot condition. After the social interaction, the more human nature traits (e.g. cognitive openness) they perceived the robot as possessing, the greater the performance improvement brought about by the presence of the robot, $b$ $=-.34,95 \% C I[-.69 ;-.08]$ (figure 4$)$.

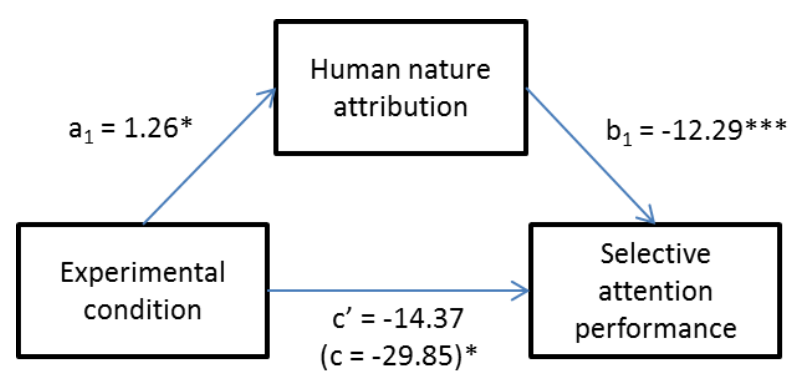

Fig. 4. Mediation of the robot presence effect on standard selective attention performance by human nature inferences.

\section{3) Warmth}

The final analysis introduced the warmth dimension as a mediator in the model, $R^{2}=.19, F(2,43)=5.11, p<.01$. As shown by the ANOVA, the participants attributed more warmth traits to the robot after the social HRI than after the non-social HRI, (a1) $t$ (44) $=2.61, p=.012,95 \%$ CI [.27; 2.12]. When the effect of warmth attribution on selective attention performance, (b1) $t(44)=-2.10, p<.041,95 \% C I$ [-16.78; -.33] was controlled for, the direct effect of the experimental conditions on selective attention performance, (c) $t(44)=-2.33, p=.025,95 \% C I[-55.72 ;-3.98]$, became non-significant, (c') $t(44)=-1.48, p=.147,95 \%$ CI $[-46.43$; 7.17], thus suggesting that mediation occurred. In sum, the participants in the social robot condition attributed more human uniqueness traits to the robot than those in the nonsocial robot condition. After the social interaction, the more uniquely human characteristics they perceived the robot as possessing, the greater the performance improvement generated by the presence of the robot, $b=-.22$, 95\%CI $[-$ $.50 ;-.01]$ (figure 4).

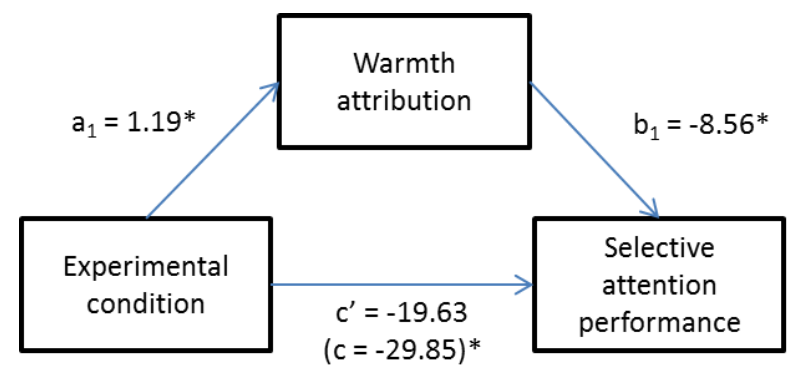

Fig. 4. Mediation of the robot presence effect on standard selective attention performance by warmth inferences.

\section{4) The best predictor of performance improvement}

We conducted a regression analysis on selective attention performance in the second session, including the scores on the 3 anthropomorphism dimensions and the 4 humanization dimensions. This analysis makes it possible to evaluate the unitary effect of each variable, while controlling for collinearity, in order to extract the best predictor. When all the other dimensions were controlled for, the results showed that only the attribution of human uniqueness traits had a significant predictive effect on selective attention performance, $b=-.63, t(45)=-2.80, p=$ $.008, \eta^{2} p=.17,95 \% C I[-25.29 ;-4.07]$. The more uniquely human traits the participants considered the robot to possess, the better they performed in the second session. We also found a statistical trend toward a significant effect of discomfort attribution: the more the participants described the robot as being characterized by discomfort traits (e.g. awkward), the lower the interference due to incongruence in 
the Flanker task was in the second session, $b=-.25, t(45)=$ $-1.75, p=.089, \eta^{2} p=.07,95 \% C I[-23.75 ; 1.75]$.

These results were confirmed by the mediation comparison analysis, including all dimensions as independent mediators in a global mediation model, $R^{2}=$ $.47, F(2,43)=4.17, p<.01$. Again, only mediation by human uniqueness was significant, $\mathrm{b}=-37,95 \% C I[-.50$; $.01]$, thereby validating the third hypothesis concerning the modulation of the robot-human conceptual distance as a mediator of SFI effects.

\section{DISCUSSION}

In the future, robots may be more present in the everyday human environment. It seems crucial to understand, today, how their presence may impact human cognitive processes. The aim of this study was to evaluate the role of the perception of robots in social presence effects on human cognitive performances resulting from the passive presence of a robot.

First, we demonstrated that social interaction changed how participants considered the robot in terms of human uniqueness (e.g., cognitive openness), human nature and warmth traits compared to a simple visual description. This result confirmed that the nature of the HRI influences humans' representations of the robot in whose presence they are [64]. It is important to note that social interaction only boosted positive attributions (i.e., more human uniqueness, human nature, warmth traits), while negative attributions (i.e., animal dehumanization, mechanistic dehumanization, discomfort traits) remained stable.

These results could point to two phenomena. First, negative attribution does not change because, unlike humans, robots seem to be considered as objects. Unlike a human being that can be dehumanized, a robot cannot be deanthropomorphized. One can only assign new human constructs [65]-[67] or social traits [30] to the robot, as if this social agent were a "blank page". However, it seems likely that this process only applies to new HRI. In the same way as for humans, it is be possible that once a robot has been anthropomorphized, subsequent HRI might modulate these inferences. However, the question of whether longterm HRI might increase, stabilize or reduce this type of anthropomorphism process remains unclear [68].

Second, these anthropomorphic inferences only occur in specific contexts that require such inferences to be made. The scientific literature shows that there are automatic neuro-socio-cognitive perceptual processes, such as face recognition (automatically activated during simple perception, even with non-humans) [69], [70]. In the case of HRI, it seems likely that the perception of and interaction with robots are based on such processes. However, this "hijacking" of these human-human interactions, together with the automatic neuro-social processes they involve and which result from the social evolution of our species, is not enough to cause the attribution of social constructs [44]. In other words, the strength of bottom-up inputs is not enough in itself. To create anthropomorphic inferences, it is necessary for the context to reinforce these bottom-up inputs through top-down processes activated by the context (e.g., the type of HRI, the robot's behavior). For example, in both experimental conditions in this study, the robot was perceived in the same way before the interaction. The robot was not seen as more "mechanical" in one condition than in the other as a function of the stability of the negative attributions. In other words, the robot started with a certain quantity of mechanical (e.g., superficial) and animal (e.g., amoral) traits and this quantity remained stable, even after the experimental induction. However, we suggest that the activation of social scripts during the social interaction would have led to the attribution of a more anthropomorphic perception of the robot [7], [13], [71]. The reason could be that when we interact with creatures (even non-humans), processes (e.g. mentalization) occur that allow us to attribute intentionality and mental activity to the other in order to understand and predict their behavior [44], [72].

We found the same effect for the "warmth" dimension of the "warmth and competence" dyad, which are considered to be universal dimensions of social perception [19]. This result is interesting in that it suggests that when people spontaneously interpret their impressions of others, these two dimensions account for almost all of the variance $(\approx$ $82 \%$ ) [73]-[75]. Given the universal qualities of the warmth and competence dimensions, it might be asked why we found no such results for competence attribution. Warmth judgments are primary, that is to say they are made before competence judgments. Warmth also carries more weight in affective and behavioral reactions. This also accounts for traits relating to de-humanization dimensions. For example, the attribution of morality traits from the human uniqueness dimension (which is strongly correlated with the warmth dimension) determines approach-avoidance tendencies that require each to evaluate the other (i.e., positively or negatively) [76]. This information is more cognitively accessible and more predictive. Competence is related more to the modulation of impression (e.g. how positive or how negative). The fact that the HRI induction in our experiment lasted only 3 minutes suggests that the participants might have gained only a general impression of the robot. Further research should focus on the evolution of these attributes in long-term HRI in comparison to human-human interaction.

Furthermore, according to our results, anthropomorphic attributions seem to mediate the effect of the presence of a social robot on selective attention performance improvement. Again, these results are in line with research on robots [43], intentional stance perception (i.e., the attribution of mental properties) [77], and the mentalization process (i.e., attribution of mental activity) [72]. In our experiment, after the social HRI, the robot could have been perceived by the participants as more conscious and similar to a fellow creature, thus resulting in a social presence effect [14]. Indeed, anthropomorphic inferences are needed if the improvement in performance is to occur in the presence of the robot. Importantly, the passive presence of the nonsocial robot during the Flanker task in the second session did not influence performance compared to the control "alone" condition. Taken together, these findings run counter to a purely mechanistic, non-social approach that reduces the effects caused by the presence of humanoid robots on attention to the action of physical distraction or interference. Indeed, with regard to the role of top-down inferences, anthropomorphism is at the center of the robot SFI processes when mechanistic distraction is controlled for.

There is evidence that when a focal task demands attentional resources, perceptual (non-social) sources of distraction can induce a conflict between the need to pay attention to the 
focal task (i.e., the central stimulus in the Flanker task) and the interfering cues (i.e., the peripheral stimuli). This conflict may result in cognitive overload and, ultimately, restrict the use of the available cues (e.g., [9]). This restriction directs the available attentional resources towards the task that is to be performed at the expense of peripheral cues (e.g. by focusing more exclusively on the central stimulus than on peripheral interfering cues). According to this view, however, both experimental conditions should have elicited the same effects, which was not the case, even though the robot behaved in a similar way during the task. Considering the mediating role of anthropomorphic inferences, we can reasonably assume that the effects of social robot presence on attention were indeed social in nature and cannot be trivialized or reduced to the action of any other nonsocial sources of distraction.

Finally, some limits have to be addressed. First, we specifically chose a mechanical humanoid robot in order to control for anthropomorphic bottom-up inferences. It is therefore not yet possible to conclude that top-down reinforcement is necessary for all situations and for all types of robots. It is possible that a sufficiently human-like robot, with fluid movements, could stimulate anthropomorphic inferences on the basis of perceptual stimuli alone [55] at the condition to avoid the "uncanny valley" phenomenon (i.e., the theory that the more similar an android robot is to a human being, the more monstrous its imperfections seem to the human observer) [55], [78]-[80]. The more human-like a robot is, the less interaction should be needed to stimulate anthropomorphism and thus social presence effects [10], [26]. The relative compatibility between the advanced technological shape and the level of perceived capacities of a robot could also play an important role [42]. Second, the social presence effect may have a negative impact on cognitive performances (i.e., social inhibition) when the amount of attentional resources needed to complete the task and the amount of attentional resources allocated to the other being present exceed an individual's reserve of cognitive resources [9]. To fully assess whether the social presence effect with robots is similar to that observed in the presence of humans, the same paradigm should be implemented with a complex task.

In conclusion, the fact that the presence of social robots can impact processes as fundamental as selective attention provides another reason to pay particular attention to the psychological, sociological, and philosophical impact of human-robot interactions.

\section{OPEN PRACTICES}

All data are publicly available via the Open Science Framework and can be accessed at https://osf.io/kfvd8/

\section{REFERENCES}

[1] R. A. Brooks, "Flesh and machines. How robots will change us.," Nelson, pp. 1-5, 2002.

[2] R. H. Paul, D. J. Libon, and R. A. Cohen, "O T O Y P O O T," Human-Computer Interact., vol. 11, no. 4, pp. 204-209, 2004.

[3] N. Riether, F. Hegel, B. Wrede, and G. Horstmann, "Social facilitation with social robots?," in Proceedings of the seventh annual ACM/IEEE international conference on Human-Robot Interaction HRI'12, 2012, p. 41.

[4] J. S. Herberg, S. Feller, I. Yengin, and M. Saerbeck, "Robot watchfulness hinders learning performance," in Proceedings - IEEE International Workshop on Robot and Human Interactive Communication, 2015, vol. 2015-Novem, pp. 153-160.
[5] N. Spatola et al., "Not as bad as it seems: When the presence of a threatening humanoid robot improves human performance," Sci. Robot., vol. 3, no. 21, p. eaat5843, 2018.

[6] N. Spatola et al., "Improved Cognitive Control in Presence of Anthropomorphized Robots," Int. J. Soc. Robot., 2019.

[7] C. Edwards, A. Edwards, P. R. Spence, and D. Westerman, "Initial Interaction Expectations with Robots: Testing the Human-To-Human Interaction Script," Commun. Stud., vol. 67, no. 2, pp. 227-238, 2016.

[8] R. B. Zajonc, "Social facilitation," Science (80-. )., vol. 149, no. 3681, pp. 269-274, 1965.

[9] R. S. Baron, "Distraction-conflict theory: Progress and problems," Adv. Exp. Soc. Psychol., vol. 19, no. C, pp. 1-40, 1986

[10] C. Nass and Y. Moon, "Machines and mindlessness: Social responses to computers," J. Soc. Issues, vol. 56, no. 1, pp. 81-103, 2000.

[11] M. W. Baldwin, "Relational schemas and the processing of social information," Psychol. Bull., vol. 112, no. 3, pp. 461-484, 1992.

[12] C. L. Stevenson, P. J. Krantz, and L. E. McClannahan, "Social interaction skills for children with autism: A script-fading procedure for nonreaders," Behav. Interv., vol. 15, no. 1, pp. 1-20, 2000.

[13] C. D. Martin, The Media Equation: How People Treat Computers, Television and New Media Like Real People and Places [Book Review], vol. 34, no. 3. Cambridge, UK: Cambridge University Press, 1997.

[14] P. Huguet, M. P. Galvaing, J. M. Monteil, and F. Dumas, "Social presence effects in the Stroop task: Further evidence for an attentional view of social facilitation," J. Pers. Soc. Psychol., vol. 77, no. 5, pp. 1011-1024, 1999.

[15] M. Augustinova and L. Ferrand, "The influence of mere social presence on Stroop interference: New evidence from the semanticallybased Stroop task," J. Exp. Soc. Psychol., vol. 48, no. 5, pp. 12131216, 2012.

[16] S. Lemaignan, J. Fink, and P. Dillenbourg, "The dynamics of anthropomorphism in robotics," Proc. 2014 ACM/IEEE Int. Conf. Human-robot Interact. - HRI '14, pp. 226-227, 2014.

[17] J. Suls, R. Martin, and L. Wheeler, "Social comparison: Why, with whom, and with what effect?," Curr. Dir. Psychol. Sci., vol. 11, no. 5, pp. 159-163, 2002.

[18] B. Scassellati, J. Kennedy, T. Belpaeme, A. Ramachandran, and F. Tanaka, "Social robots for education: A review," Sci. Robot., vol. 3, no. 21 , p. eaat5954, 2018.

[19] S. T. Fiske, A. J. C. Cuddy, and P. Glick, "Universal dimensions of social cognition: warmth and competence," Trends Cogn. Sci., vol. 11 , no. 2 , pp. 77-83, 2007.

[20] N. Haslam, "Dehumanization: An integrative review," Personality and Social Psychology Review, vol. 10, no. 3. pp. 252-264, 2006.

[21] B. A. Eriksen and C. W. Eriksen, "Effects of noise letters upon the identification of a target letter in a nonsearch task," Percept. Psychophys., vol. 16, no. 1, pp. 143-149, 1974

[22] G. Dreisbach and S. Böttcher, "How the social-evaluative context modulates processes of cognitive control," Psychol. Res., vol. 75, no. 2, pp. 143-151, 2011.

[23] S. Atmaca, N. Sebanz, and G. Knoblich, "The joint flanker effect: Sharing tasks with real and imagined co-actors," Exp. Brain Res., vol. 211, no. 3-4, pp. 371-385, 2011.

[24] C. D. Martin, "The Media Equation: How People Treat Computers, Television and New Media Like Real People and Places [Book Review]," IEEE Spectrum, vol. 34, no. 3. pp. 9-10, 1997.

[25] I. Kollar, F. Fischer, and F. W. Hesse, "Collaboration scripts - A conceptual analysis," Educ. Psychol. Rev., vol. 18, no. 2, pp. 159$185,2006$.

[26] E.-L. Sallnäs, K. Rassmus-Gröhn, and C. Sjöström, Supporting presence in collaborative environments by haptic force feedback, vol. 7, no. 4. 2002

[27] C. Breazeal, "Social interactions in HRI: The robot view," IEEE Trans. Syst. Man Cybern. Part C Appl. Rev., vol. 34, no. 2, pp. 181186,2004

[28] K. Tanaka, H. Nakanishi, and H. Ishiguro, "Comparing Video, Avatar, and Robot Mediated Communication: Pros and Cons of Embodiment," in Collaboration Technologies and Social Computing, 2014, vol. 460, pp. 96-110.

[29] T. Chaminade, J. Hodgins, and M. Kawato, "Anthropomorphism influences perception of computer-animated characters' actions," Soc. Cogn. Affect. Neurosci., vol. 2, no. 3, pp. 206-216, 2007.

[30] C. M. Carpinella, A. B. Wyman, M. A. Perez, and S. J. Stroessner, "The Robotic Social Attributes Scale (RoSAS)," in Proceedings of the 2017 ACM/IEEE International Conference on Human-Robot Interaction - HRI '17, 2017, pp. 254-262.

[31] N. Spatola et al., "National stereotypes and robots' perception: the 'made in' effect," Front. Robot. AI, 2019. 
[32] S. T. Fiske, "Stereotype Content: Warmth and Competence Endure," Curr. Dir. Psychol. Sci., 2018

[33] J.-C. Croizet, J.-P. Leyens, V. Yzerbyt, and G. Schadron, "Stereotypes and Social Cognition.," Contemp. Sociol., vol. 25, no. 3, p. 402,2006

[34] N. Haslam and S. Loughnan, "Dehumanization and Infrahumanization," 2014.

[35] C. H. Dupree and S. T. Fiske, "Universal dimensions of social signals: Warmth and competence," in Social Signal Processing, 2017, pp. 23-33.

[36] D. Kuchenbrandt, F. Eyssel, S. Bobinger, and M. Neufeld, "When a Robot's Group Membership Matters: Anthropomorphization of Robots as a Function of Social Categorization," Int. J. Soc. Robot., vol. 5, no. 3, pp. 409-417, 2013.

[37] J. Shi, Y. Kashima, S. Loughnan, C. Suitner, and N. Haslam, "Subhuman, Inhuman, and Superhuman: Contrasting Humans with Nonhumans in Three Cultures," Soc. Cogn., vol. 26, no. 2, pp. 248 $258,2008$.

[38] J.-P. Leyens, S. Demoulin, R. Gaunt, M. P. Paladino, and J. Vaes, "Infra-humanization: The Wall of Group Differences," Soc. Issues Policy Rev., vol. 1, no. 1, pp. 139-172, 2010.

[39] S. T. Fiske, "Attention and weight in person perception: The impact of negative and extreme behavior," Soc. Cogn. Sel. Work. Susan Fisk., vol. 82, no. 6, pp. 15-40, 2018.

[40] L. T. Harris, S. M. McClure, W. Van Den Bos, J. D. Cohen, and S. T. Fiske, "Regions of the MPFC differentially tuned to social and nonsocial affective evaluation," Cogn. Affect. Behav. Neurosci., vol. 7, no. 4, pp. 309-316, 2007.

[41] E. Oztop, T. Chaminade, and D. W. Franklin, "Human-humanoid interaction: is a humanoid robot perceived as a human?," IEEE/RAS Int. Conf. Humanoid Robot., vol. 2, no. 4, pp. 830-841, 2004.

[42] T. Chaminade, D. W. Franklin, E. Oztop, and G. Cheng, "Motor interference between humans and humanoid robots: Effect of biological and artificial motion," in Proceedings of 2005 4th IEEE International Conference on Development and Learning, 2005, vol. 2005, pp. 96-101.

[43] T. Chaminade et al., "Brain response to a humanoid robot in areas implicated in the perception of human emotional gestures," PLoS One, vol. 5, no. 7, 2010.

[44] T. Chaminade et al., "How do we think machines think? An fMRI study of alleged competition with an artificial intelligence," Front. Hum. Neurosci., vol. 6, 2012.

[45] G. Rizzolatti, "The mirror neuron system and its function in humans," in Anatomy and Embryology, 2005, vol. 210, no. 5-6, pp. 419-421.

[46] K. L. Bunday, R. N. Lemon, J. M. Kilner, M. Davare, and G. A. Orban, "Grasp-specific motor resonance is influenced by the visibility of the observed actor," Cortex, 2016.

[47] V. Gallese and A. Goldman, "Mirror neurons and the simulation theory of mind-reading," Trends in Cognitive Sciences, vol. 2, no. 12. pp. 493-501, 1998.

[48] R. A. Zwaan and L. J. Taylor, "Seeing, acting, understanding: Motor resonance in language comprehension," J. Exp. Psychol. Gen., vol. 135, no. 1, pp. 1-11, 2006.

[49] A. P. Saygin, "Point-Light Biological Motion Perception Activates Human Premotor Cortex," J. Neurosci., vol. 24, no. 27, pp. 61816188,2004

[50] M. E. W. Varnum, C. Blais, and G. A. Brewer, "Social class affects Mu-suppression during action observation," Soc. Neurosci., 2016.

[51] E. Cracco, L. De Coster, M. Andres, and M. Brass, "Mirroring multiple agents: Motor resonance during action observation is modulated by the number of agents," Soc. Cogn. Affect. Neurosci., 2016.

[52] S. M. Aglioti, P. Cesari, M. Romani, and C. Urgesi, "Action anticipation and motor resonance in elite basketball players," Nat. Neurosci., vol. 11, no. 9, pp. 1109-1116, 2008.

[53] N. Epley, A. Waytz, and J. T. Cacioppo, "On Seeing Human: A Three-Factor Theory of Anthropomorphism," Psychol. Rev., vol. 114, no. 4, pp. 864-886, 2007.

[54] A. Wykowska, T. Chaminade, and G. Cheng, "Embodied artificial agents for understanding human social cognition," Philosophical Transactions of the Royal Society B: Biological Sciences. 2016.

[55] A. P. Saygin, T. Chaminade, H. Ishiguro, J. Driver, and C. Frith, "The thing that should not be: Predictive coding and the uncanny valley in perceiving human and humanoid robot actions," Soc. Cogn. Affect. Neurosci., vol. 7, no. 4, pp. 413-422, 2012.

[56] M. Salem, F. Eyssel, K. Rohlfing, S. Kopp, and F. Joublin, "To Err is Human(-like): Effects of Robot Gesture on Perceived Anthropomorphism and Likability," Int. J. Soc. Robot., vol. 5, no. 3, pp. 313-323, 2013.
[57] A. M. Rosenthal-Von Der Pütten et al., "Neural correlates of empathy towards robots," in ACM/IEEE International Conference on HumanRobot Interaction, 2013, pp. 215-216.

[58] L. D. Riek, T.-C. Rabinowitch, B. Chakrabarti, and P. Robinson, "How anthropomorphism affects empathy toward robots," in Proceedings of the 4th ACM/IEEE international conference on Human robot interaction - HRI '09, 2009, p. 245

[59] J. Mulholland, Review: The Media Equation: How People Treat Computers, Television and New Media like Real People and Places, vol. 113, no. 1. 2018.

[60] F. Eyssel and D. Kuchenbrandt, "Manipulating anthropomorphic inferences about NAO: The role of situational and dispositional aspects of effectance motivation," in Proceedings - IEEE International Workshop on Robot and Human Interactive Communication, 2011, pp. 467-472.

[61] C. R. Wilson Van Voorhis and B. L. Morgan, "Understanding Power and Rules of Thumb for Determining Sample Sizes," Tutor. Quant. Methods Psychol., vol. 3, no. 2, pp. 43-50, 2007.

[62] F. Faul, E. Erdfelder, A. G. Lang, and A. Buchner, "G*Power 3: A flexible statistical power analysis program for the social, behavioral, and biomedical sciences," Behav. Res. Methods, vol. 39, no. 2, pp. 175-191, 2007.

[63] B. Hanington and B. Martin, Universal methods of design: 100 ways to research complex problems, develop innovative ideas, and design effective solutions, vol. 49, no. 10. Rockport Publishers, 2012.

[64] F. Hegel, S. Krach, T. Kircher, B. Wrede, and G. Sagerer, "Understanding social robots: A user study on anthropomorphism," in Proceedings of the 17th IEEE International Symposium on Robot and Human Interactive Communication, RO-MAN, 2008, pp. 574-579.

[65] B. Tay, Y. Jung, and T. Park, "When stereotypes meet robots: The double-edge sword of robot gender and personality in human-robot interaction," Comput. Human Behav., vol. 38, pp. 75-84, 2014.

[66] F. Eyssel and F. Hegel, "(S)he's Got the Look: Gender Stereotyping of Robots," J. Appl. Soc. Psychol., vol. 42, no. 9, pp. 2213-2230, 2012.

[67] F. Eyssel and D. Kuchenbrandt, "Social categorization of social robots: Anthropomorphism as a function of robot group membership," Br. J. Soc. Psychol., vol. 51, no. 4, pp. 724-731, 2012.

[68] J. M. K. Westlund, H. W. Park, R. Williams, and C. Breazeal, "Measuring young children's long-term relationships with social robots," in Proceedings of the 17th ACM Conference on Interaction Design and Children - IDC '18, 2018, pp. 207-218.

[69] D. S. Berry and J. L. Finch Wero, "Accuracy in Face Perception: A View from Ecological Psychology," J. Pers., vol. 61, no. 4, pp. 497520, 1993.

[70] A. Stigliani, K. Grill-Spector, V. S. Natu, K. S. Weiner, and J. Gomez, "The functional neuroanatomy of face perception: from brain measurements to deep neural networks," Interface Focus, vol. 8, no. 4, p. 20180013, 2018.

[71] F. Van Overwalle, "Social cognition and the brain: A meta-analysis," Human Brain Mapping, vol. 30, no. 3. pp. 829-858, 2009.

[72] E. Koh, Affect regulation, mentalization, and the development of the self, vol. 38, no. 1-2. New York, NY: Other Press, 2004.

[73] B. Wojciszke, R. Bazinska, and M. Jaworski, "On the dominance of moral categories in impression formation," Personal. Soc. Psychol. Bull., vol. 24, no. 12, pp. 1251-1263, 1998.

[74] B. Wojciszke, "Morality and competence in person- and selfperception," Eur. Rev. Soc. Psychol., vol. 16, no. 1, pp. 155-188, 2005.

[75] B. Wojciszke, "Affective concomitants of information on morality and competence," Eur. Psychol., vol. 10, no. 1, pp. 60-70, 2005.

[76] J. T. Cacioppo, W. L. Gardner, and G. G. Berntson, "Beyond bipolar conceptualizations and measures: The case of attitudes and evaluative space," Personal. Soc. Psychol. Rev., vol. 1, no. 1, pp. 3-25, 1997.

[77] S. Gupta, "Intentional Systems," Corp. Capital. Polit. Philos., vol. 68, no. 4, pp. 52-62, 2017.

[78] J. Seyama and R. S. Nagayama, "The uncanny valley: Effect of realism on the impression of artificial human faces," Presence Teleoperators Virtual Environ., vol. 16, no. 4, pp. 337-351, 2007.

[79] K. F. MacDorman and H. Ishiguro, "The uncanny advantage of using androids in cognitive and social science research," Interact. Stud., vol. 7, no. 3, pp. 297-337, 2006.

[80] M. Mori, K. F. MacDorman, and N. Kageki, "The uncanny valley," IEEE Robot. Autom. Mag., vol. 19, no. 2, pp. 98-100, 2012. 